Article

\title{
Aminopeptidase Expression in Multiple Myeloma Associates with Disease Progression and Sensitivity to Melflufen
}

\author{
Juho J. Miettinen ${ }^{1,+} \mathbb{D}^{-}$, Romika Kumari ${ }^{1,+}{ }^{,}$Gunnhildur Asta Traustadottir ${ }^{2}{ }^{\oplus}$, Maiju-Emilia Huppunen ${ }^{1}$, \\ Philipp Sergeev ${ }^{1}$, Muntasir M. Majumder ${ }^{1}$, Alexander Schepsky ${ }^{2}{ }^{\circledR}$, Thorarinn Gudjonsson ${ }^{2} \mathbb{D}$, Juha Lievonen ${ }^{3}$, \\ Despina Bazou ${ }^{4}$, Paul Dowling ${ }^{5}$, Peter $\mathrm{O}^{\prime}$ Gorman ${ }^{4}$, Ana Slipicevic ${ }^{6}$, Pekka Anttila ${ }^{3}$, Raija Silvennoinen ${ }^{3}(\mathbb{D}$, \\ Nina N. Nupponen ${ }^{6}$, Fredrik Lehmann ${ }^{6}$ and Caroline A. Heckman ${ }^{1, * \mathbb{D}}$
}

1 Institute for Molecular Medicine Finland-FIMM, HiLIFE-Helsinki Institute of Life Science, iCAN Digital Precision Cancer Medicine Flagship, University of Helsinki, 00290 Helsinki, Finland; juho.miettinen@helsinki.fi (J.J.M.); romika.kumari@helsinki.fi (R.K.); maiju-emilia.huppunen@helsinki.fi (M.-E.H.); philipp.sergeev@helsinki.fi (P.S.); muntasir.mamun@helsinki.fi (M.M.M.)

2 Stem Cell Research Unit, Biomedical Center, University of Iceland, 101 Reykjavik, Iceland; gutra@hi.is (G.A.T.); schepsky@hi.is (A.S.); tgudjons@hi.is (T.G.)

3 Department of Hematology, Helsinki University Hospital Comprehensive Cancer Center, 00290 Helsinki, Finland; juha.lievonen@hus.fi (J.L.); pekka.anttila@hus.fi (P.A.); raija.silvennoinen@helsinki.fi (R.S.)

Citation: Miettinen, J.J.; Kumari, R.; Traustadottir, G.A.; Huppunen, M.-E.; Sergeev, P.; Majumder, M.M.; Schepsky, A.; Gudjonsson, T.; Lievonen, J.; Bazou, D.; et al. Aminopeptidase Expression in Multiple Myeloma Associates with Disease Progression and Sensitivity to Melflufen. Cancers 2021, 13, 1527. https://doi.org/10.3390/cancers 13071527

Academic Editor: Enrique M. Ocio

Received: 8 January 2021

Accepted: 20 March 2021

Published: 26 March 2021

Publisher's Note: MDPI stays neutral with regard to jurisdictional claims in published maps and institutional affiliations.

Copyright: (c) 2021 by the authors. Licensee MDPI, Basel, Switzerland. This article is an open access article distributed under the terms and conditions of the Creative Commons Attribution (CC BY) license (https:// creativecommons.org/licenses/by/ $4.0 /)$.
4 Department of Hematology, Mater Misericordiae University Hospital, D07 Dublin, Ireland; despina.bazou@ucd.ie (D.B.); pogorman@mirtireland.com (P.O.)

5 Department of Biology, Maynooth University, National University of Ireland, W23 F2H6 Maynooth, Co. Kildare, Ireland; paul.dowling@mu.ie

6 Oncopeptides AB, 11153 Stockholm, Sweden; ana.slipicevic@oncopeptides.com (A.S.); nina.nupponen@oncopeptides.com (N.N.N.); fredrik.lehmann@oncopeptides.com (F.L.)

* Correspondence: caroline.heckman@helsinki.fi; Tel.: +358-50-415-6769

$\dagger$ These authors equally contributed to this work.

Simple Summary: The aims of this study were to investigate aminopeptidase expression in multiple myeloma and to identify the aminopeptidases responsible for the activation of the peptide-drug conjugate melflufen in multiple myeloma. We observed a differential expression of aminopeptidases between relapsed/refractory and newly diagnosed multiple myeloma patients. A higher expression of the aminopeptidase genes XPNPEP1, RNPEP, DPP3, and BLMH in multiple myeloma plasma cells was associated with shorter patient overall survival. The peptide-drug conjugate melflufen was particularly active towards plasma cells from relapsed/refractory multiple myeloma patients. Melflufen could be hydrolyzed to its active form by the aminopeptidases LAP3, LTA4H, RNPEP, and ANPEP, all of which are expressed in multiple myeloma. These results indicate critical roles for aminopeptidases in disease progression and the activity of melflufen in multiple myeloma.

Abstract: Multiple myeloma (MM) is characterized by extensive immunoglobulin production leading to an excessive load on protein homeostasis in tumor cells. Aminopeptidases contribute to proteolysis by catalyzing the hydrolysis of amino acids from proteins or peptides and function downstream of the ubiquitin-proteasome pathway. Notably, aminopeptidases can be utilized in the delivery of antibody and peptide-conjugated drugs, such as melflufen, currently in clinical trials. We analyzed the expression of 39 aminopeptidase genes in MM samples from 122 patients treated at Finnish cancer centers and 892 patients from the CoMMpass database. Based on ranked abundance, LAP3, ERAP2, METAP2, TTP2, and DPP7 were highly expressed in MM. ERAP2, XPNPEP1, DPP3, RNPEP, and CTSV were differentially expressed between relapsed/refractory and newly diagnosed MM samples $(p<0.05)$. Sensitivity to melflufen was detected ex vivo in 11/15 MM patient samples, and high sensitivity was observed, especially in relapsed/refractory samples. Survival analysis revealed that high expression of XPNPEP1, RNPEP, DPP3, and BLMH $(p<0.05)$ was associated with shorter overall survival. Hydrolysis analysis demonstrated that melflufen is a substrate for aminopeptidases LAP3, LTA4H, RNPEP, and ANPEP. The sensitivity of MM cell lines to melflufen 
was reduced by aminopeptidase inhibitors. These results indicate critical roles of aminopeptidases in disease progression and the activity of melflufen in MM.

Keywords: multiple myeloma; aminopeptidase; gene expression; melflufen

\section{Introduction}

Multiple myeloma (MM) is a highly heterogeneous disease with complex genetic alterations. Advanced molecular profiling of MM has revealed novel therapeutic targets that may aid in drug development. Nevertheless, MM is still incurable for the majority of patients, and new therapeutic approaches are required to improve patient survival. One of the hallmarks of MM is the excessive production of abnormal immunoglobulins, which makes the myeloma cells heavily dependent on the ubiquitin-proteasome system to maintain protein homeostasis and availability of free amino acids. In general, human tumors are highly dependent on free amino acids for their growth, with disruption of protein turnover shown to induce apoptotic cascades in MM as well as in acute myeloid leukemia (AML) [1]. Due to this dependency, inhibition of the ubiquitin-proteasome system is currently the standard of care and a widely accepted strategy in the treatment of both newly diagnosed (NDMM) and relapsed/refractory multiple myeloma (RRMM) patients [2].

Aminopeptidases are an important group of metalloenzymes that catalyze the hydrolysis of terminal amino acid residues from proteins or peptides and operate as the last step downstream of the ubiquitin-proteasome system. Consequently, they are implicated in several cellular functions, including development, differentiation, cell cycle, DNA repair, signal transduction, and programmed cell death [3]. Around 40 genes encoding aminopeptidases have been identified in the human genome, and their activity in the human bone marrow (BM) and blood cells has been described as early as the 1960s $[4,5]$. Aminopeptidases are involved in many human disorders, including autoimmune diseases and cancer. For example, aminopeptidase N (ANPEP alias CD13) is abnormally expressed in AML, B-cell acute lymphoblastic leukemia, and chronic lymphoblastic leukemia, where it may be used as a diagnostic or prognostic marker [6-8]. ANPEP is also expressed in a subset of Waldenström macroglobulinemia, MM, and plasma cell leukemias [9,10]. ANPEP expression in samples from MM patients has been shown to correlate with shorter overall survival [11,12]. Recently, endoplasmic reticulum aminopeptidase 2 (ERAP2) expression has been associated with a better outcome in patients with bladder cancer treated with immunotherapies [13]. Dipeptidyl peptidase 8 (DPP8) has recently been described as a novel target for MM and AML therapeutics [14,15].

Even though aminopeptidases have conserved structures and their role in diseases is well recognized, there are relatively few therapeutic molecules directly targeting these enzymes. Bestatin was the first aminopeptidase inhibitor used in clinical settings, while more recently, a new-generation inhibitor, tosedostat (CHR-2797), entered in clinical trials for AML and myelodysplastic syndromes [16,17]. An alternative approach to directly targeting aminopeptidases is to exploit their mode of action in cells. This is based on observations that aminopeptidase expression may differ between normal and malignant cells or even among different subcellular compartments within a cell. The effects of antibody-drug conjugates or peptide-drug conjugates, such as melphalan flufenamide (hereinafter named melflufen), are facilitated by protease/peptidase hydrolysis, which leads to the release of a highly toxic payload inside the target cells $[18,19]$. Aminopeptidases have been shown to be one of the peptidase groups facilitating melflufen activation in cells [19]. Melflufen has been shown to have antitumor activity against MM cell lines and primary MM cells [20,21].

To further understand the role of aminopeptidases in $\mathrm{MM}$ and disease progression, we investigated the expression of aminopeptidase gene family members in a large cohort 
of samples from MM patients and assessed differences in expression between NDMM and RRMM. Furthermore, we determined whether specific aminopeptidases are associated with poor prognosis in patients with MM. Using BM cells from MM patients, we observed remarkable ex vivo sensitivity of MM plasma cells to melflufen, particularly in samples from RRMM patients. We were also able to demonstrate that melflufen is a substrate of leucine aminopeptidase 3 (LAP3), leukotriene A4 hydrolase (LTA4H), arginyl aminopeptidase (RNPEP), and ANPEP, which we also found to be expressed in MM.

\section{Materials and Methods}

\subsection{Sample Collection}

Patient samples were collected after informed consent and using protocols approved by an ethical committee of the Helsinki University Hospital (permit numbers 239/13/03/00/ 2010,303/13/03/01/2011) in accordance with the Declaration of Helsinki. In total, 178 (NDMM, $n=57$; RRMM, $n=121$ ) different patient samples were collected from 140 different MM patients (Table 1, Online Supplementary Table S1). Bone marrow mononuclear cells (BMMNCs) were isolated from BM aspirates by Ficoll-Paque gradient centrifugation (GE Healthcare, Little Chalfont, Buckinghamshire, UK). For RNA and exome sequencing, BM CD138+ plasma cells were enriched by immunomagnetic bead selection (StemCell Technologies, Vancouver, BC, Canada).

Table 1. MM patient and Disease Characteristics and Treatment History in the FIMM Dataset.

\begin{tabular}{|c|c|c|c|}
\hline \multicolumn{4}{|c|}{ Patient and disease characteristics by disease stage ${ }^{a}$} \\
\hline & $\operatorname{NDMM}(n=57)$ & RRMM $(n=83)$ & Total $(n=140)$ \\
\hline Age at diagnosis, years, median (range) & $65(46-84)$ & $63(26-81)$ & $64(26-84)$ \\
\hline Sex, female/male, $n$ & $27 / 30$ & $31 / 52$ & $58 / 82$ \\
\hline \multicolumn{4}{|l|}{ Cytogenetics, $n(\%)$} \\
\hline $\mathrm{t}(11 ; 14)$ & $15(26.3)$ & $16(19.3)$ & $31(22.1)$ \\
\hline $\mathrm{t}(4 ; 14)$ & $9(15.8)$ & $19(22.9)$ & $28(20.0)$ \\
\hline $\mathrm{t}(14 ; 16)$ & $2(3.5)$ & $2(2.4)$ & $4(2.9)$ \\
\hline $\mathrm{t}(14 ; 20)$ & 0 & $2(2.4)$ & $2(1.4)$ \\
\hline $\operatorname{del}(17 p)$ & $5(8.8)$ & $20(24.1)$ & $25(17.9)$ \\
\hline $\operatorname{del}(13 q) /-13$ & $39(68.4)$ & $42(50.6)$ & $81(57.9)$ \\
\hline 1qgain & $18(31.6)$ & $46(55.4)$ & $64(45.7)$ \\
\hline No abnormalities found & $2(3.5)$ & 0 & $2(1.4)$ \\
\hline \multicolumn{4}{|l|}{ International Staging System, $n(\%)$} \\
\hline 1 & $11(19.3)$ & $19(22.9)$ & $30(21.4)$ \\
\hline 2 & $27(47.4)$ & $23(27.7)$ & $50(35.7)$ \\
\hline 3 & $11(19.3)$ & $16(19.3)$ & $27(19.3)$ \\
\hline Not available & $8(14.0)$ & $25(30.1)$ & $33(23.6)$ \\
\hline \multicolumn{4}{|c|}{ Treatment history of relapsed/refractory patients $(n=83)$} \\
\hline Prior treatment, $n(\%)$ & Exposed & Refractory & Not exposed \\
\hline Alkylating agents (MEL, CPM) & $63(75.9)$ & $16(19.3)$ & $4(4.8)$ \\
\hline Bortezomib & $44(53.0)$ & $28(33.7)$ & $11(13.3)$ \\
\hline IMiDs & $31(37.3)$ & $34(41.0)$ & $18(21.7)$ \\
\hline
\end{tabular}

a If a patient provided both NDMM and RRMM samples, this patient was included in the NDMM group. If a patient provided samples at multiple relapse stages and the diagnosis sample was missing, then data from the first relapse were included in the table. FIMM: Institute for Molecular Medicine Finland; NDMM: newly diagnosed multiple myeloma; RRMM: relapsed/refractory multiple myeloma; MEL: melphalan; CPM: cyclophosphamide; IMiDs: immunomodulatory drugs.

\subsection{RNA Sequencing and Analysis}

RNA was extracted from CD138+ plasma cells using the AllPrep ${ }^{\circledR}$ DNA/RNA/miRNA Universal or miRNeasy kits (Qiagen, Hilden, Germany). RNA integrity was measured on an Agilent Bioanalyzer 2100 instrument (Agilent, Santa Clara, CA, USA); only samples with RNA integrity $\geq 7$ were used for sequencing. Illumina-compatible RNA sequenc- 
ing libraries were prepared using ScriptSeq ${ }^{\mathrm{TM}}$ technology and sequenced on Illumina HiSeq ${ }^{\circledR} 1500$ or 2500 instruments (Illumina, San Diego, CA, USA). After preprocessing, filtered reads were aligned to the GRCh38 human reference genome using the STAR aligner tool [22]. Gene read counts were normalized using the reads per kilobase of transcript per million mapped reads (RPKM) method. In total, 39 annotated aminopeptidase genes (Online Supplementary Table S2) were identified in the human genome (assembly GRCh38) utilizing the Ensembl release 99 and NCBI databases by using the search term "aminopeptidase" and further confirming the molecular function (gene ontology) of identified genes. A cutoff value $>1$ RPKM was used to filter the expressed aminopeptidase genes. The DESeq2 tool was used to identify differentially expressed genes in samples from newly diagnosed multiple myeloma (NDMM) vs. relapsed/refractory multiple myeloma (RRMM) [23].

The association of aminopeptidase gene expression with survival outcome was estimated by Kaplan-Meier analysis; the analysis was performed using expression-based grouping of the samples, whereby samples were grouped into "high" ( $\geq$ median expression) and "low" (<median expression) expression groups. The significance of the difference between the two groups (high vs. low expression) was deduced using a Mantel-Cox logrank test.

\subsection{Data Validation Using the CoMMpass Dataset}

To validate our results, clinical, gene expression, and genomic variant data (somatic mutation and copy number variation (CNVs)) were obtained from the Multiple Myeloma Research Foundation (MMRF) Relating Clinical Outcomes in MM to Personal Assessment of Genetic Profile (CoMMpass) study (both https://research.themmrf.org and www.themmrf.org, were accessed on 5 February 2018).

The MMRF CoMMpass gene expression dataset included 892 samples in total $(87 \%$ baseline/diagnosis, $12 \%$ progressive disease, and 1\% missing): 875 bone marrow samples and 17 peripheral blood samples. The MMRF CoMMpass samples were obtained at baseline/diagnosis $(n=780)$, or from patients with progressive disease $(n=81)$, stable disease $(n=12)$, partial response $(n=7)$, and very good partial response $(n=4)$ to treatment; for 8 patients, disease information was missing. A total of 1044 samples (bone marrow, $n=1021$; peripheral blood, $n=23$ ) were used for CNV analysis; samples corresponded to baseline $(n=877)$, progressive disease $(n=90)$, partial response $(n=17)$, stable disease $(n=14)$, very good partial response $(n=9)$, complete response $(n=4)$, and stringent complete response $(n=1)$; information was missing for 32 samples. A total of 1164 samples (bone marrow, $n=1140$; peripheral blood, $n=24$ ) were included in the somatic mutation dataset; samples corresponded to baseline $(n=946)$, progressive disease $(n=122)$, stable disease $(n=20)$, partial response $(n=20)$, very good partial response $(n=16)$, and complete response $(n=6)$; information was missing for 34 samples.

\subsection{Exome Sequencing and Cytogenetics}

The DNeasy ${ }^{\circledR}$ Blood \& Tissue kit or AllPrep ${ }^{\circledR}$ DNA/RNA/miRNA Universal kit (Qiagen) was used to isolate genomic DNA from skin biopsies and CD138+ cells. The SeqCap ${ }^{\circledR}$ EZ MedExome kit (Roche NimbleGen, Madison, WI), SureSelect Clinical Research Exome kit, or SureSelect Human All Exon V5 kit (Agilent Technologies, Santa Clara, CA) was used for exome capture. Sequencing was performed on a HiSeq ${ }^{\circledR} 1500$ or 2500 instrument. VarScan2 somatic algorithm was implemented for calling somatic mutations [24], and mutation annotations were performed using SnpEff 4.04 [25]. Gene CNVs were identified using the CopyCat tool (https:/ / github.com/chrisamiller/copycat, accessed on 19 September 2014). Cytogenetic data were generated using routine diagnostic fluorescence in situ hybridization technology following European Myeloma Network 2012 guidelines [26].

\subsection{Liquid Chromatography-Tandem Mass Spectrometry-Based Proteomics}

BM-MNC CD138+ cells from 23 MM patient samples were lysed in RIPA buffer (Cell Signaling Technology, Danvers, MA, USA), and proteins digested. An amount of $500 \mathrm{ng}$ 
of each digested whole-cell protein lysate was loaded onto a Q Exactive (Thermo Fisher Scientific, Hemel Hempstead, UK) high-resolution accurate mass spectrometer connected to a Dionex Ultimate 3000 (RSLCnano) chromatography system (Thermo Fisher Scientific, Hemel Hempstead, UK). Peptides were separated using a 2\% to $40 \%$ gradient of acetonitrile on a Biobasic C18 Picofrit column (Thermo Fisher Scientific, Hemel Hempstead, UK) (100 mm length, $75 \mathrm{~mm}$ internal diameter) for over $65 \mathrm{~min}$ at a flow rate of $250 \mathrm{~nL} / \mathrm{min}$. Data were acquired with the mass spectrometer (MS) operating in automatic data-dependent switching mode. A full MS scan at 140,000 resolution and a range of $300-1700 \mathrm{~m} / \mathrm{z}$ was followed by an MS/MS scan at 17,500 resolution and a range of 200-2000 m/z, selecting the 10 most intense ions prior to MS/MS.

\subsection{Proteomics Data Analysis}

Protein identification and label-free quantification normalization of MS/MS data were performed using MaxQuant v1.5.2.8 (http:/ / www.maxquant.org, accessed on 20 May 2015). The Andromeda search algorithm incorporated in the MaxQuant software was used to correlate MS/MS data against the Homo sapiens UniProt reference proteome database (release 2016_11) and a contaminant sequence set provided by MaxQuant. Perseus v.1.5.6.0 (www.maxquant.org/, accessed on 1 June 2016) was used for data analysis, processing, and visualization. Normalized label-free quantification intensity values were used as the quantitative measurement of protein abundance for subsequent analysis. The data matrix was filtered for the removal of contaminants and peptides identified by site. Label-free quantification intensity values were $\log 2$ transformed.

\subsection{Flow Cytometry-Based Drug Sensitivity Testing}

For flow cytometry (FC)-based drug testing, selinexor, 4-hydroperoxycyclophosphamide, and bortezomib (Online Supplementary Table S3) were dispensed into 96-well V-bottom plates (Thermo Fisher Scientific, Carlsbad, CA, USA) using an acoustic liquid handling device, Echo 550 (Labcyte, Sunnyvale, CA, USA). Melflufen and melphalan were manually pipetted into the 96-well V-bottom plate wells. Drug plate layout and concentrations are presented in Online Supplementary Figure S1. Viably frozen BM-MNCs were thawed, freezing solution washed away, cell pellet suspended in conditioned medium (RPMI 1640 medium supplemented with $10 \%$ fetal bovine serum (FBS), $2 \mathrm{mM} \mathrm{L-glutamine,} \mathrm{peni-}$ cillin $(100 \mathrm{U} / \mathrm{mL})$, streptomycin $(100 \mu \mathrm{g} / \mathrm{mL})$, and $25 \%$ conditioned medium from the HS-5 human bone marrow stromal cell line), DNase I (Promega, Madison, WI, USA) treated for $60 \mathrm{~min}$, and cultured in conditioned medium overnight. Cells were filtered through a $70 \mu \mathrm{m}$ cell strainer (Fisher Scientific, Pittsburg, PA), cell viability measured, and cells plated in parallel on pre-drugged 96-well plates $(100,000$ viable cells/well in $100 \mu \mathrm{L})$. If cell viability was poor $(<50 \%)$, the Dead Cell Removal kit (Miltenyi Biotec, Auburn, CA, USA) was used prior to plating the cells. Stock solutions $(10,000 \mu \mathrm{M})$ of melflufen (Recipharm $\mathrm{AB}$, Stockholm, Sweden) and melphalan (Sigma-Aldrich, St. Louis, MO, USA) in DMSO, which had been prepared in advance and stored at $-80^{\circ} \mathrm{C}$, were thawed and diluted in conditioned medium to desired concentrations shortly before the drug testing to minimize autohydrolysis of melflufen and melphalan (exchange of $\mathrm{Cl}$ with $\mathrm{OH}$ at the $\mathrm{N}$-mustard part of the molecules). Melflufen and melphalan were added to the 96 -well plates by manually pipetting only after the cells had been plated, just prior to the start of the drug sensitivity test. The cells were incubated with the drugs for $72 \mathrm{~h}$ at $37{ }^{\circ} \mathrm{C}$ and $5 \% \mathrm{CO}_{2}$. Following $72 \mathrm{~h}$ incubation with the drugs, the cells were centrifuged $(500 \times \mathrm{g}, 6 \mathrm{~min})$ in the 96-well plates, and media discarded by inverting the plate. The cells were suspended in $25 \mu \mathrm{L}$ of antibody mix containing staining buffer (5\% FBS in Dulbecco's phosphate-buffered saline) and the following two antibodies (BD Biosciences; Santa Jose, CA, USA): CD138 (BV605, clone MI15, dilution 1:100) and CD38 (BV786, clone HIT2, dilution 1:100). The cells were stained for $30 \mathrm{~min}$ at room temperature in the dark and subsequently washed with $100 \mu \mathrm{L}$ staining buffer followed by centrifugation $(500 \times g, 6 \mathrm{~min})$ and supernatant removal by inverting the plate. Apoptotic and dead cells were discriminated by 7 -aminoactinomycin 
D (7-AAD) and PE Annexin V (BD Biosciences) staining with both dyes diluted 1:50 in $25 \mu \mathrm{L}$ annexin $\mathrm{V}$ binding buffer. The plates were incubated for $10 \mathrm{~min}$ at room temperature before FC analysis. FC analysis was performed using the IntelliCyt iQue Screener PLUS instrument (Sartorius, Goettingen, Germany). MM patient sample BM-MNCs were acquired for analysis from each 96-well plate well by the iQue Screener PLUS instrument using a $17 \mathrm{~s}$ sip time per well and a pump speed of 32 revolutions per minute, resulting in 35 min reading time for a full 96-well plate. ForeCyt software (Sartorius) was used to gate cells and acquire population counts. Analysis was done from viable singlet cells, and the detailed gating strategy is illustrated in Online Supplementary Figure S2. The cell count of a well was normalized to its six adjacent DMSO controls, from which the highest and lowest values were excluded and normalized values were used for calculating cell viability percentages for each tested drug concentration, and dose-response curves drawn. Half-maximal effective concentration (EC50) values were calculated based on the dose-response curves.

\subsection{Cell Lines and Viability Assay}

RPMI-8226 (ATCC ${ }^{\circledR}$ CCL-155 ${ }^{\mathrm{TM}}$ ) and MM.1S (ATCC ${ }^{\circledR}$ CRL-2974 ${ }^{\mathrm{TM}}$ ) cell lines were purchased from the American Type Culture Collection (ATCC; Wesel, Germany). Both cell lines were cultured in RPMI-1640 medium (Gibco, Thermo Fisher Scientific, Waltham, MA, USA), supplemented with $10 \%$ heat-inactivated fetal bovine serum (FBS; Gibco, Thermo Fisher Scientific; Waltham, MA, USA), $100 \mathrm{U} / \mathrm{mL}$ penicillin, and $100 \mu \mathrm{g} / \mathrm{mL}$ streptomycin (Gibco, Thermo Fisher Scientific, Waltham, MA, USA) at $37^{\circ} \mathrm{C}$ in a humidified incubator with $5 \% \mathrm{CO}_{2}$.

The resazurin-based PrestoBlue cell viability reagent (Thermo Fisher Scientific, Waltham, MA, USA) was used to assess the viability of RPMI-8226 and MM.1S. Cells were treated for $48 \mathrm{~h}$ with complete growth medium supplemented with $3.75,7.5,15,30$, or $60 \mu \mathrm{M}$ tosedostat. Then, RPMI-8226 and MM.1S cells were pretreated with DMSO (control) or the metalloaminopeptidase inhibitors bestatin $(10 \mu \mathrm{M})$ and tosedostat $(10 \mu \mathrm{M})$ for $1 \mathrm{~h}$, and then either left without additional treatment or treated with three different concentrations $(1,3$, and $5 \mu \mathrm{M})$ of melflufen/melphalan for $15 \mathrm{~min}$, after which the medium was replaced. Cells were cultured in 96 -well culture plates at a density of 20,000 cells/well ( 250 cells $/ \mu \mathrm{L})$, incubated for $48 \mathrm{~h}$ at $37^{\circ} \mathrm{C}$ in a humidified incubator with $5 \% \mathrm{CO}_{2}$, after PrestoBlue was added (1/10 th of the total volume). After $2 \mathrm{~h}$ incubation at $37^{\circ} \mathrm{C}$ in a humidified incubator with $5 \% \mathrm{CO}_{2}$, absorbance was read at $570 \mathrm{~nm}$ with a reference wavelength of $595 \mathrm{~nm}$. Each experimental setup was performed three times independently with at least three replicates, and values were normalized to untreated control (mean \pm standard deviation $(\mathrm{SD}), n=3)$. Statistical significance was assessed with one- or two-way analysis of variance (ANOVA) followed by Dunnett's or Tukey's multiple comparison tests using GraphPad Prism $\left({ }^{*} p \leq 0.05,{ }^{* *} p \leq 0.01,{ }^{* * *} p \leq 0.001,{ }^{* * * *} p \leq 0.0001\right)$.

\subsection{Hydrolysis Assay}

Frozen solutions containing the aminopeptidases LAP3 (TP309052) (OriGene, Rockville, MD, USA), DPP3 (8087), DPP7 (3438-SE), LTA4H (4008-ZN), RNPEP (8089-ZN), XNPEP1 (2970-ZN), and ANPEP (3815-ZN) (R\&D Systems, Minneapolis, MN, USA) were thawed and diluted with their incubation buffers (Online Supplementary Table S4) to the desired concentrations. A stock solution $(1000 \mu \mathrm{M})$ of the test substrate melflufen was prepared in DMSO and further diluted in incubation buffer to the desired concentration shortly before the experiment to minimize autohydrolysis of melflufen. Incubation buffer and substrates were mixed and incubated with aminopeptidases for $2 \mathrm{~h}$ at a substrate-to-enzyme ratio of 5000:1. Control incubations were performed without aminopeptidases. All incubations were performed in duplicate at $37^{\circ} \mathrm{C}$. The incubation was stopped by adding ice-cold acetonitrile. The samples were centrifuged and diluted with eluent $\mathrm{A}$ (water + formic acid, $1000+1, v+v)$. The samples were subjected to liquid chromatography-high-resolution mass spectrometry analysis. The analysis system consisted of a binary high-performance 
liquid chromatography (HPLC) pump (1290 Series, Agilent), a column oven at $40{ }^{\circ} \mathrm{C}$ (1260 series, Agilent), an autosampler (HTC PAL, CTC Analytics, Zwingen, Switzerland), and an Orbitrap mass spectrometer with electrospray ionization (Q Exactive, Thermo Scientific). A Phenomenex Luna $3 \mu \mathrm{C} 18(2)$ HPLC column $(50 \times 2 \mathrm{~mm}, 3 \mu \mathrm{m})$ was used with a C18 $4 \times 2 \mathrm{~mm}$ guard column (Phenomenex, Torrance, CA), and a binary gradient of eluents $\mathrm{A}$ and $\mathrm{B}$ (acetonitrile + formic acid, $1000+1, \mathrm{v}+\mathrm{v}$ ) was applied for separation. Mass spectra were recorded in positive ionization mode at a resolution of 35,000 in FullScan mode. The MS data were evaluated for $(\mathrm{M}+\mathrm{M})^{+}$traces of melflufen, monohydroxy melphalan flufenamide, dihydroxy melphalan flufenamide, and 4-F-PheOEt, with peaks integrated using LCquan (Thermo Scientific).

\section{Results}

\subsection{Differential Expression of Aminopeptidase Genes in $M M$}

To determine relative mRNA expression patterns of aminopeptidases in MM cells, we performed RNAseq analysis on $122 \mathrm{MM}$ patient-derived samples (NDMM $=41$, RRMM = 81) from 99 different patients (Online Supplementary Table S1). First, we ranked aminopeptidase gene expression levels based on mRNA abundance in all samples and observed that most aminopeptidases were expressed (Figure 1A, Table 2, Online Supplementary Table S5). The most abundantly expressed aminopeptidase genes were $L A P 3, E R A P 2$, methionyl aminopeptidase 2 (METAP2), tripeptidyl peptidase 2 (TTP2), DPP7, ERAP1, $L T A 4 H$, and leucyl and cystinyl aminopeptidase (LNPEP) with a median log2(RPKM) range of 5.00 (LAP3) to 2.93 (LNPEP). The least abundantly expressed aminopeptidase genes were thyrotropin-releasing hormone-degrading enzyme (TRHDE), $\mathrm{F}$ coagulation factor XI (F11), X-prolyl aminopeptidase 2 (XPNPEP2), cathepsin V (CTSV), laeverin (LVRN), and archaelysin family metallopeptidase 1 (AMZ1) with a median log2(RPKM) range of -6.57 (TRDHE) to -3.60 (AMZ1). We further verified that aminopeptidase gene expression profiles are comparable between the Multiple Myeloma Research Foundation (MMRF) CoMMpass dataset $(n=892)$ and our internal (FIMM) dataset with a correlation coefficient of $0.93\left(p=2.2 \times 10^{-16}\right)$ (Online Supplementary Figure S3). In the FIMM dataset, aminopeptidases clustered hierarchically into four subgroups, with group I displaying the highest level of gene expression and group IV the lowest (Figure 1B). We observed correlation between expression levels of some aminopeptidase genes in group I, for example, TPP2 and $D P P 7$, and certain cytogenetic markers and with patient age (Online Supplementary Figure S4). TPP2 was expressed at a lower level in patients with del $13 q$ and in patients $\geq 65$ years of age. DPP7 was expressed at a lower level in patients with either $t(4 ; 14)$ or $t(11 ; 14)$, but was more highly expressed in patients $\geq 65$ years of age.

Liquid chromatography-tandem mass spectrometry-based proteomics data were collected from 23 MM patients ( $n=14$ NDMM; $n=9$ RRMM) (Online Supplementary Table S1). The proteomic results showed a positive correlation with mRNA expression in CD138+ cells enriched from MM patient BM-MNCs. Out of the 39 aminopeptidases, peptides positively identifying 17 of these proteolytic enzymes were detected by mass spectrometry (Online Supplementary Figure S5A, Table S6). Of these 17 aminopeptidases, six, namely, LAP3, bleomycin hydrolase (BLMH), DPP3, DPP7, RNPEP, and ERAP2, showed the highest correlation between protein and gene expression levels (Online Supplementary Figure S5B).

\subsection{Association between Aminopeptidase Gene Expression and MM Disease Status}

Next, we investigated whether the expression of aminopeptidase genes could be linked to disease status. We compared aminopeptidase gene expression profiles between the 41 NDMM and 81 RRMM patient samples in the FIMM dataset. In total, five aminopeptidases, ERAP2, XPNPEP1, DPP3, RNPEP, and CTSV, were differentially expressed in the RRMM and NDMM samples $(p<0.05$; adjusted $p<0.1$ ), indicating potential roles in disease progression (Figure 2A). Of the five differentially expressed aminopeptidase genes, only ERAP2 was decreased in RRMM vs. NDMM samples (Figure 2B). In the MMRF CoMMpass 
dataset using paired samples (NDMM, $n=39$; RRMM, $n=45$ ), similar differences were observed, although only CTSV had an adjusted $p$-value $<0.1$ (Online Supplementary Figure S6). We observed correlation between the expression levels of some of the five differentially expressed aminopeptidase genes in RRMM vs. NDMM, for example, DPP3, $R N P E P$, and XPNPEP1, and certain cytogenetic markers and with International Staging System (ISS) stage (Online Supplementary Figure S7A,B). DPP3 was expressed at a lower level in patients with $\mathrm{t}(11 ; 14)$, and also in ISS stage 2 compared with ISS stage 3 . RNPEP was more highly expressed in patients with either del 13q or $\mathrm{t}(4 ; 14)$ or 1q gain, and in ISS stage 3 compared with both ISS stages 1 and 2. XPNPEP1 was more highly expressed in patients with 1q gain, and in ISS stage 3 compared with ISS stage 2.


Figure 1. Expression of aminopeptidase gene family in MM patient CD138+ cells. (A) Boxplot showing the log2(RPKM) expression of 39 aminopeptidase and 17 housekeeping genes from $122 \mathrm{MM}$ samples (NDMM $(n=41), \operatorname{RRMM}(n=81))$ taken from 99 different patients. Genes are ranked based on median expression values. (B) Heatmap showing the hierarchical clustering of aminopeptidase gene expression, with group I (blue) containing genes with the highest and group IV (magenta) containing genes with the lowest level of expression. Groups II (red) and III (green) contain genes with an intermediate level of expression. Sample cytogenetics (del 13q, del 17p, $\mathrm{t}(4 ; 14), \mathrm{t}(11 ; 14), 1 \mathrm{q}$ gain) are indicated above the heatmap. Disease stage indicates whether the sample was from NDMM (shown as cyan; samples on the left) or RRMM (pink; samples on the right). RPKM: reads per kilobase of transcript per million mapped reads; MM: multiple myeloma; NDMM: newly diagnosed multiple myeloma; RRMM: relapsed/refractory multiple myeloma. 
Table 2. List of evaluated aminopeptidase genes $(n=39)$, sorted by median log2(RPKM) expression from RNAseq data from 122 multiple myeloma patient samples in the FIMM dataset.

\begin{tabular}{|c|c|c|c|c|}
\hline Gene Symbol a & Genomic Location & $\begin{array}{l}\text { Median } \log 2(\mathrm{RPKM}) \\
\text { Expression }(n=122)\end{array}$ & Gene Name ${ }^{a}$ & $\begin{array}{l}\text { Peptidase/Protein } \\
\text { Family }\end{array}$ \\
\hline$L A P 3$ & 4p15.32 & 5.00 & Leucine aminopeptidase 3 & M \\
\hline ERAP2 & $5 q 15$ & 4.40 & $\begin{array}{l}\text { Endoplasmic reticulum } \\
\text { aminopeptidase } 2\end{array}$ & M \\
\hline METAP2 & $12 q 22$ & 4.22 & Methionyl aminopeptidase 2 & M \\
\hline TPP2 & $13 q 33.1$ & 4.13 & Tripeptidyl peptidase 2 & $\mathrm{~S}$ \\
\hline$D P P 7$ & $9 q 34.3$ & 4.04 & Dipeptidyl peptidase 7 & $S$ \\
\hline ERAP1 & $5 q 15$ & 4.03 & $\begin{array}{l}\text { Endoplasmic reticulum } \\
\text { aminopeptidase } 1\end{array}$ & M \\
\hline LTA4H & $12 \mathrm{q} 23.1$ & 3.44 & Leukotriene A4 hydrolase & $\mathrm{M}$ \\
\hline LNPEP & $5 q 15$ & 2.93 & $\begin{array}{l}\text { Leucyl and cystinyl } \\
\text { aminopeptidase }\end{array}$ & M \\
\hline XPNPEP1 & $10 \mathrm{q} 25.1$ & 2.66 & X-prolyl aminopeptidase 1 & M \\
\hline METAP1 & $4 q 23$ & 2.46 & Methionyl aminopeptidase 1 & M \\
\hline DPP3 & $11 \mathrm{q} 13.2$ & 2.28 & Dipeptidyl peptidase 3 & M \\
\hline$D P P 8$ & $15 q 22.31$ & 2.21 & Dipeptidyl peptidase 8 & S \\
\hline NPEPPS & $17 q 21.32$ & 2.12 & $\begin{array}{l}\text { Aminopeptidase puromycin } \\
\text { sensitive }\end{array}$ & M \\
\hline$B L M H$ & $17 q 11.2$ & 1.83 & Bleomycin hydrolase & $\mathrm{C}$ \\
\hline JMJD7 & $15 q 15.1$ & 1.78 & Jumonji domain containing 7 & * \\
\hline RNPEP & $1 \mathrm{q} 32.1$ & 1.76 & Arginyl aminopeptidase & M \\
\hline PGPEP1 & 19p13.11 & 1.72 & Pyroglutamyl-peptidase I & $\mathrm{C}$ \\
\hline TPP1 & $11 \mathrm{p} 15.4$ & 1.59 & Tripeptidyl peptidase 1 & $S$ \\
\hline PEPD & $19 q 13.11$ & 1.41 & Peptidase D & M \\
\hline DNPEP & $2 q 35$ & 1.16 & Aspartyl aminopeptidase & $\mathrm{M}$ \\
\hline DPP9 & $19 \mathrm{p} 13.3$ & 1.16 & Dipeptidyl peptidase 9 & $S$ \\
\hline METAP1D & $2 q 31.1$ & 0.38 & $\begin{array}{l}\text { Methionyl aminopeptidase } \\
\text { type } 1 \mathrm{D} \text {, mitochondrial }\end{array}$ & M \\
\hline NPEPL1 & $20 q 13.32$ & 0.37 & Aminopeptidase like 1 & M \\
\hline $\mathrm{CTSH}$ & $15 q 25.1$ & 0.36 & Cathepsin $\mathrm{H}$ & $\mathrm{C}$ \\
\hline XPNPEP3 & $22 \mathrm{q} 13.2$ & -0.04 & X-prolyl aminopeptidase 3 & M \\
\hline RNPEPL1 & $2 q 37.3$ & -0.1 & Arginyl aminopeptidase like 1 & M \\
\hline AOPEP & $9 q 22.32$ & -0.38 & Aminopeptidase $\mathrm{O}$ (putative) & M \\
\hline KDM8 & $16 \mathrm{p} 12.1$ & -1.3 & Lysine demethylase 8 & $*$ \\
\hline$A N P E P$ & $15 q 26.1$ & -2.13 & $\begin{array}{l}\text { Alanyl aminopeptidase, } \\
\text { membrane }\end{array}$ & M \\
\hline ENPEP & $4 q 25$ & -2.15 & Glutamyl Aminopeptidase & M \\
\hline MMP14 & $14 \mathrm{q} 11.2$ & -2.83 & Matrix Metallopeptidase 14 & M \\
\hline$D P P 4$ & $2 q 24.2$ & -2.84 & Dipeptidyl Peptidase 4 & $\mathrm{~S}$ \\
\hline NAALADL1 & 11q13.1 & -3.39 & $\begin{array}{l}\text { N-Acetylated Alpha-Linked } \\
\text { Acidic Dipeptidase Like } 1\end{array}$ & M \\
\hline$A M Z 1$ & $7 \mathrm{p} 22.3$ & -3.6 & $\begin{array}{l}\text { Archaelysin Family } \\
\text { Metallopeptidase } 1\end{array}$ & M \\
\hline$L V R N$ & $5 q 23.1$ & -3.64 & Laeverin & M \\
\hline CTSV & $9 q 22.33$ & -4.39 & Cathepsin V & $\mathrm{C}$ \\
\hline XPNPEP2 & Xq26.1 & -6.15 & X-Prolyl Aminopeptidase 2 & M \\
\hline$F 11$ & $4 \mathrm{q} 35.2$ & -6.22 & Coagulation Factor XI & $\mathrm{S}$ \\
\hline TRHDE & $12 q 21.1$ & -6.57 & $\begin{array}{l}\text { Thyrotropin Releasing } \\
\text { Hormone Degrading Enzyme }\end{array}$ & M \\
\hline
\end{tabular}

a Source of gene symbols and names: the HUGO Gene Nomenclature Committee (HGNC); RPKM: reads per kilobase of transcript per million mapped reads; C: cysteine peptidase; M: metallopeptidase; S: serine peptidase; *: Jumonji oxygenase family. 



Figure 2. Aminopeptidase genes are differentially expressed in NDMM versus RRMM. (A) Five aminopeptidase genes (ERAP2, XPNPEP1, DPP3, RNPEP, CTSV) were differentially expressed in RRMM $(n=82)$ and NDMM $(n=41)$ samples $(p<0.05$; adjusted $p<0.1)$ in the FIMM dataset. Differential gene expression between NDMM and RRMM was determined using the DESeq2 tool. (B) Among these five genes, only the expression level of ERAP2 was decreased in RRMM versus NDMM samples. NDMM: newly diagnosed multiple myeloma; RRMM: relapsed/refractory multiple myeloma; RPKM: reads per kilobase of transcript per million mapped reads; ap: adjusted $p$-value.

\subsection{Prognostic Significance of Aminopeptidase Gene Expression}

High expression of four aminopeptidase genes associated with significantly poorer prognosis in MM patients from both FIMM (Figure 3A-E and Online Supplementary Figure S8A-F) and CoMMpass datasets (Online Supplementary Figure S9A-M). The median overall survival associated with patient samples showing high versus low gene expression was 55 vs. 122 months for XPNPEP1 (hazard ratio (HR), 3.263; 95\% confidence limit (CL), 1.749-6.087; $p=0.00012$ ) (Figure 3B), 73 vs. 100 months for RNPEP (HR, 2.271; $95 \%$ CL, $1.248-4.129 ; p=0.0062$ ) (Figure 3C), 73 vs. 120 months for DPP3 (HR, 1.956; 95\% $\mathrm{CL}, 1.108-3.453 ; p=0.02)$ (Figure 3D), and 68 vs. 122 months for BLMH (HR, 1.815; 95\% CL, 1.035-3.184; $p=0.037$ ) (Figure 3E, Online Supplementary Table S7).

\subsection{Aminopeptidase Somatic Mutation and CNV Characteristics in Myeloma}

Exome sequence analysis of 169 samples ( $n=56$ NDMM and $n=113$ RRMM) from 132 different MM patients in the FIMM dataset (Online Supplementary Table S1) indicated that small somatic variants within the protein coding regions of aminopeptidase genes are relatively rare. In this dataset, NPEPPS was mutated in $2.37 \%$ of the samples $(4 / 169), L V R N$ in $2.37 \%$ of the samples $(4 / 169)$, and BLMH in $1.78 \%$ of the samples $(3 / 169)$, while the frequency of mutations in other aminopeptidases was below 1.2\% (Online Supplementary Figure S10, Table S8). Mutation frequencies were similarly infrequent in the CoMMpass dataset samples $(n=1164)$, with aminopeptidase mutations identified in less than $1.2 \%$ of the samples (Online Supplementary Figure S11). 

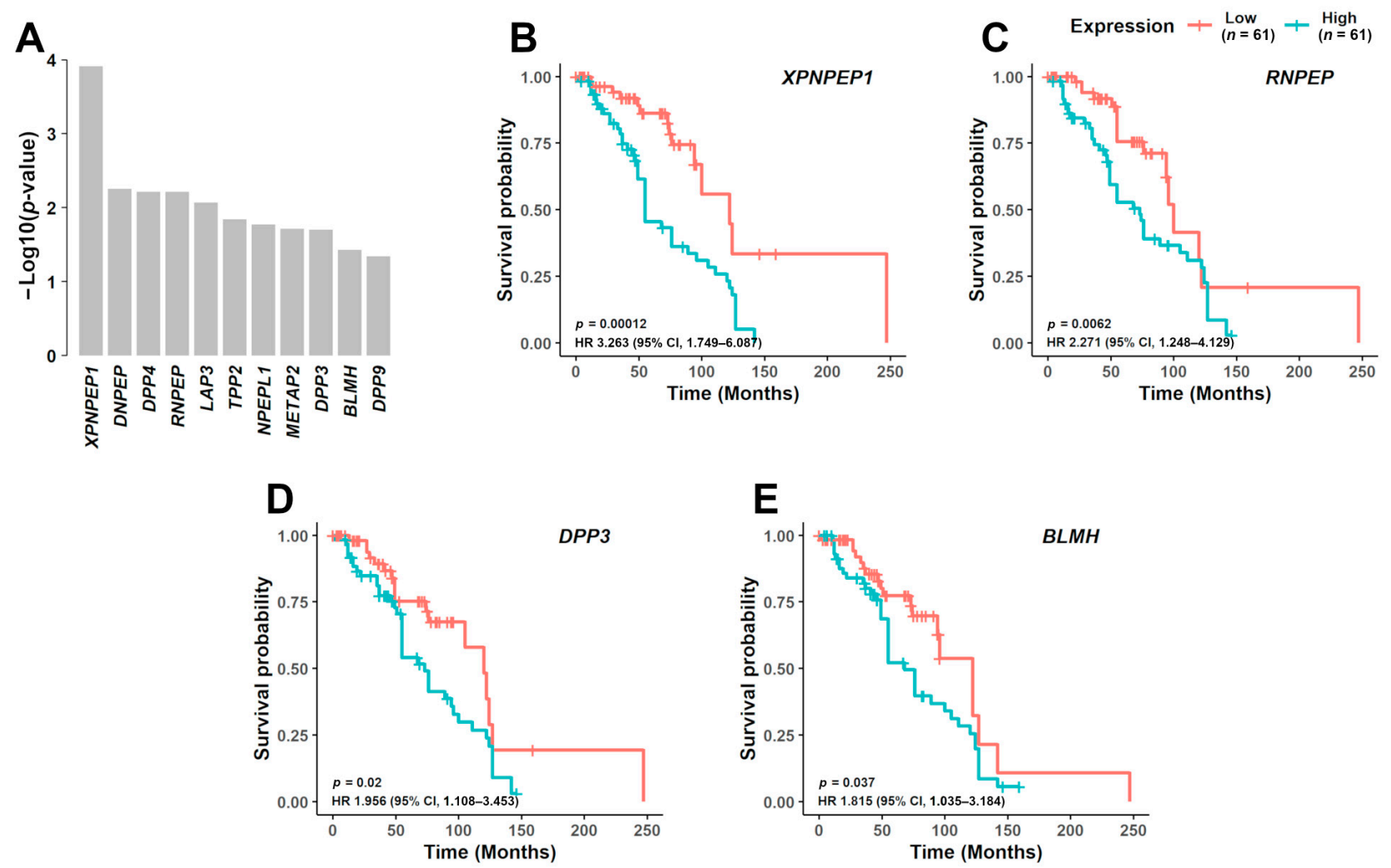

Figure 3. Prognostic significance of aminopeptidase gene expression in MM. (A) In the FIMM dataset consisting of $122 \mathrm{MM}$ samples, NDMM $(n=41)$ and RRMM $(n=81)$, from 99 different patients, 11 aminopeptidase genes were predicted to be associated with poor prognosis $(p<0.05)$. From these 11 genes, 4 were further validated in the CoMMpass dataset. Survival curves are shown for the 4 aminopeptidase genes based on the FIMM dataset, dividing the patients with low gene expression $(n=61)$ and high gene expression $(n=61)$ : (B) XPNPEP1, (C) RNPEP, (D) DPP3, and (E) BLMH. NDMM: newly diagnosed multiple myeloma; RRMM: relapsed/refractory multiple myeloma; HR: hazard ratio; CI: confidence interval.

$\mathrm{CNV}$ analysis showed that gains in the copy number of the aminopeptidase genes DPP9 (31.95\%), PGPEP1 (27.22\%), RNPEP (20.12\%), PEPD (20.12\%), and DPP7 (17.75\%) were frequent, whereas deletions of TPP2 (40.24\%), XPNPEP2 (13.61\%), and XPNPEP3 $(8.87 \%)$ were observed in more than $8 \%$ of the samples (Online Supplementary Figure S12A, Figure S13, Table S9). Results in the FIMM dataset were validated using the CoMMpass dataset: the correlation coefficient for aminopeptidase gain percentage was 0.73 , and for aminopeptidase deletion, the percentage was 0.7 (Online Supplementary Figure S12B). As expected, RRMM samples exhibited a higher number of CNVs associated with aminopeptidase genes than NDMM samples (RRMM: $n=467$ overall, with gains in 317 and deletions in 150 vs. NDMM: $n=159$, with gains in 95 and deletions in 64).

\subsection{Patient Bone Marrow CD138+CD38+ Plasma Cells Are Sensitive to Melflufen}

Aminopeptidases not only have a role in disease progression, but these enzymes are required to direct the activity of peptide-drug conjugates, such as melflufen. To determine whether melflufen activity can be detected and differs in BM samples from MM patients, we evaluated the efficacy of melflufen and four additional drugs (melphalan, selinexor, bortezomib, and 4-hydroperoxycyclophosphamide) towards CD138+CD38+ plasma cells in $15 \mathrm{BM}-\mathrm{MNC}$ samples (NDMM = 6; RRMM = 9) obtained from 14 different MM patients (Online Supplementary Figure S14, Tables S1). CD138+CD38+ plasma cell-specific responses to the drugs were measured by multiplexed high-throughput FC. The fraction of CD138+CD38+ cells from all live cells in the tested samples varied between $0.4 \%$ and $27.9 \%$ after $72 \mathrm{~h}$ incubation in the ex vivo culturing conditions without drug treatment (Online Supplementary Table S10). Of the 5 drugs tested, melflufen showed the highest activity 
against CD138+CD38+ cells (median EC50 = $0.9 \mathrm{nM})($ Online Supplementary Table S10). Of the 15 samples tested, 5 were highly sensitive to melflufen (median EC50 $<0.1 \mathrm{nM}$ ), 6 were intermediately sensitive (median EC50 0.1-5 nM), and the 4 least-sensitive samples had a median EC50 $>5 \mathrm{nM}$ (Figure 4A). In general, CD138+CD38+ cells were more sensitive to melflufen than melphalan (median EC50 = $1473 \mathrm{nM}$ ) (Online Supplementary Table S11). There was a positive correlation between melflufen and melphalan response in CD138+CD38+ cells $(r=0.66 ; p=0.009)$ (Figure 4B). We did not observe correlation between myeloma patient sample CD138+CD38+ cell melflufen sensitivity and aminopeptidase gene expression in a small set of 10 myeloma samples (Online Supplementary Figures S15 and S16, Table S12).

A

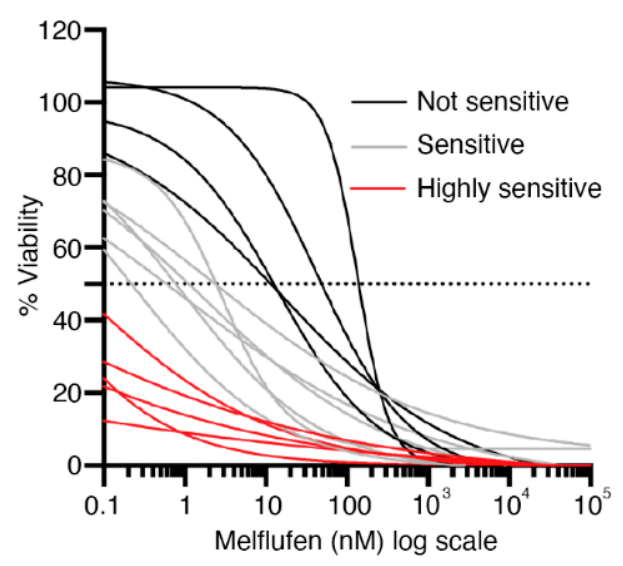

B

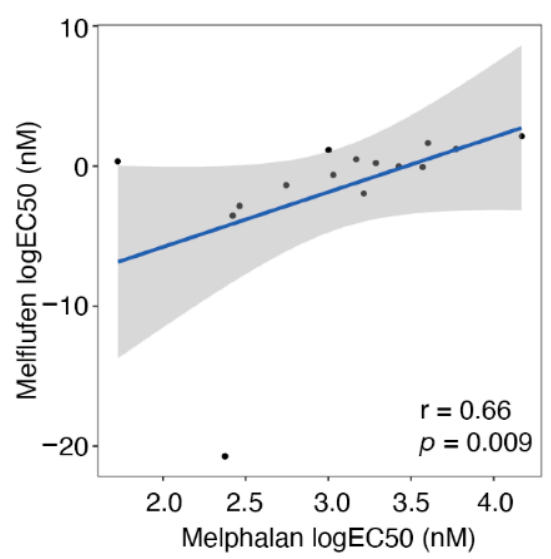

C

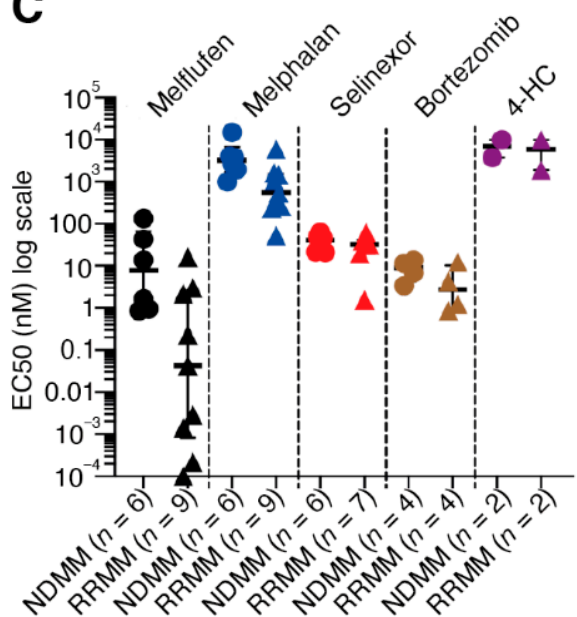

Figure 4. MM patient bone marrow CD138+CD38+ plasma cells are sensitive to melflufen. (A) Drug sensitivity was measured after $72 \mathrm{~h}$ incubation with melflufen or with DMSO alone (control). Cell viability was assessed by multicolor high-throughput flow cytometry using annexin V and 7AAD viability markers. The viability (\%) of CD138+CD38+ cells was calculated, and dose-response curves drawn. The MM patient samples can be divided into three groups based on the sensitivity of their CD138+CD38+ cells to melflufen: highly sensitive (red: EC50<0.1 nM), intermediately sensitive (gray: EC50 0.1-5 nM), not sensitive (black: EC50 > $5 \mathrm{nM}$ ). (B) Correlation of CD138+CD38+ cell sensitivity to melflufen ( $y$-axis) with melphalan sensitivity ( $x$-axis). Drug sensitivity was measured using EC50 values. (C) Comparison of CD138+CD38+ cell sensitivity (EC50) between NDMM (dots) and RRMM (triangles) in patient samples treated with melflufen (black; $p=0.0004$ ), melphalan (blue; $p=0.025$ ), selinexor (red; $p=0.44$ ), bortezomib (brown; $p=0.34$ ), and 4-HC (purple; $p=1$ ). DMSO: dimethyl sulfoxide; 4-HC: 4-hydroperoxycyclophosphamide; NDMM: newly diagnosed multiple myeloma; RRMM: relapsed/refractory multiple myeloma.

Of the other tested drugs, selinexor, a XPO1/CRM1 inhibitor, also showed high toxicity against CD138+CD38+ cells (median EC50 = 35.4 nM) (Online Supplementary Figure S14, Table S11). MM sample sensitivity to selinexor did not correlate with melflufen sensitivity or any of the other tested drugs (Online Supplementary Figure S17A-C). Bortezomib, a proteasome subunit S26 inhibitor, showed a very narrow window of response in CD138+CD38+ cells in the ex vivo assay with a median EC50 = 5.7 nM (Online Supplementary Figure S14, Table S11). There were five samples that could be considered sensitive to bortezomib (median EC50 = $4.2 \mathrm{nM}$ ) and three samples insensitive (median EC50 = $12.3 \mathrm{nM}$ ). None of the samples showed sensitivity to 4-hydroperoxycyclophosphamide, the active metabolite of cyclophosphamide, with an EC50 $\geq 1900$ nM in the ex vivo assay (Online Supplementary Figure S14, Table S11). 


\subsection{RRMM Samples Are More Sensitive to Melflufen than NDMM Samples}

Interestingly, our FC-based drug sensitivity testing results suggested that CD138+CD38+ cells from RRMM patients were more sensitive ex vivo to melflufen compared with CD138+CD38+ cells in NDMM samples (RRMM median EC50 = $0.04 \mathrm{nM}$; NDMM median $\mathrm{EC} 50=7.7 \mathrm{nM} ; p=0.0004$ ) (Figure $4 \mathrm{C}$ ). A similar trend was observed with melphalan, although the median EC50 values were clearly higher (RRMM median EC50 = 556 nM; NDMM median EC50 = $3193 \mathrm{nM} ; p=0.025$ ) when compared with melflufen (Figure 4C). CD138+CD38+ cells from patients with poor prognosis indicators, such as cytogenetic markers del $17 \mathrm{p}$ and $\mathrm{t}(4 ; 14)$, included both melflufen-sensitive $(n=5)$ and insensitive $(n=5)$ samples (Online Supplementary Figure S18). No clear biomarkers for sensitivity to melflufen were identified based on cytogenetic markers alone. Both melflufen-sensitive and insensitive samples expressed aminopeptidase genes (Figure 1B).

\subsection{Melflufen Activity Is Dependent on Aminopeptidase Activity}

To determine whether melflufen activity is dependent on aminopeptidase activity, MM cell lines RPMI-8226 and MM.1S were first treated with the metalloaminopeptidase inhibitors bestatin and tosedostat, then treated with melflufen or melphalan, and the viability of cells measured after $48 \mathrm{~h}$. The viability of RPMI-8226 and MM.1S cells decreased in a dose-dependent manner upon $48 \mathrm{~h}$ treatment with tosedostat alone (Figure 5A). Treating RPMI-8226 cells with $10 \mu \mathrm{M}$ tosedostat prior to treatment with melflufen significantly increased the viability of the cells from $60.41 \%$ to $78.15 \%$ with $3 \mu \mathrm{M}$ melflufen $(p=0.0086)$ and from $21.77 \%$ to $54.55 \%$ with $5 \mu \mathrm{M}$ melflufen ( $p \leq 0.0001$ ), compared with untreated controls (Figure 5B). Likewise, treating MM.1S cells with $10 \mu \mathrm{M}$ tosedostat prior to treatment with melflufen significantly increased the viability of the cells from $39.82 \%$ to $73.78 \%$ for $0.5 \mu \mathrm{M}$ melflufen $(p=0.0175)$ and from $19.81 \%$ to $51.60 \%$ for $1 \mu \mathrm{M}$ melflufen $(p=0.0046)$ compared with untreated control cells (Figure 5C). In addition, treating RPMI-8226 and MM.1S cells with bestatin reduced their sensitivity to melflufen, albeit not significantly.

\subsection{Aminopeptidases LAP3, LTA4H, RNPEP, DPP7, and ANPEP Hydrolyze Melflufen}

Melflufen can be hydrolyzed to melphalan and para-fluoro-L-phenylalanine ethyl ester (4-F-Phe-OEt) under suitable conditions (Figure 6A, Online Supplementary Figure S19, Online Supplementary Table S4). We tested seven aminopeptidases, DPP3, DPP7, XPNPEP1, LAP3, LTA4H, RNPEP, and ANPEP, to determine whether they induce melflufen amide hydrolysis in vitro. The release of 4-F-Phe-OEt from melflufen was measured after incubating melflufen with the aminopeptidases for $2 \mathrm{~h}$. Release of 4-F-Phe-OEt was measured using liquid chromatography-high-resolution mass spectrometry, which showed that the peak area of melflufen decreases while the peak area for 4-F-Phe-OEt increases when a specific aminopeptidase is added. A clear increase of 4-F-Phe-OEt was observed after melflufen incubation with aminopeptidases LAP3 (Figure 6B), LTA4H (Figure 6C), RNPEP (Figure 6D), and ANPEP (Online Supplementary Figure S19), suggesting that these aminopeptidases can hydrolyze melflufen. However, no such increase was observed with DPP3 and XPNPEP1. Interestingly, aminopeptidase DPP7 induced hydrolysis of the terminal ester and not the amide of the melflufen molecule. The DPP family of enzymes hydrolyzes the peptide bond between two amino acids from the N-terminal. Due to the structure of melflufen, it is likely that DPP7 hydrolyzes the ester rather than the amide bond. 

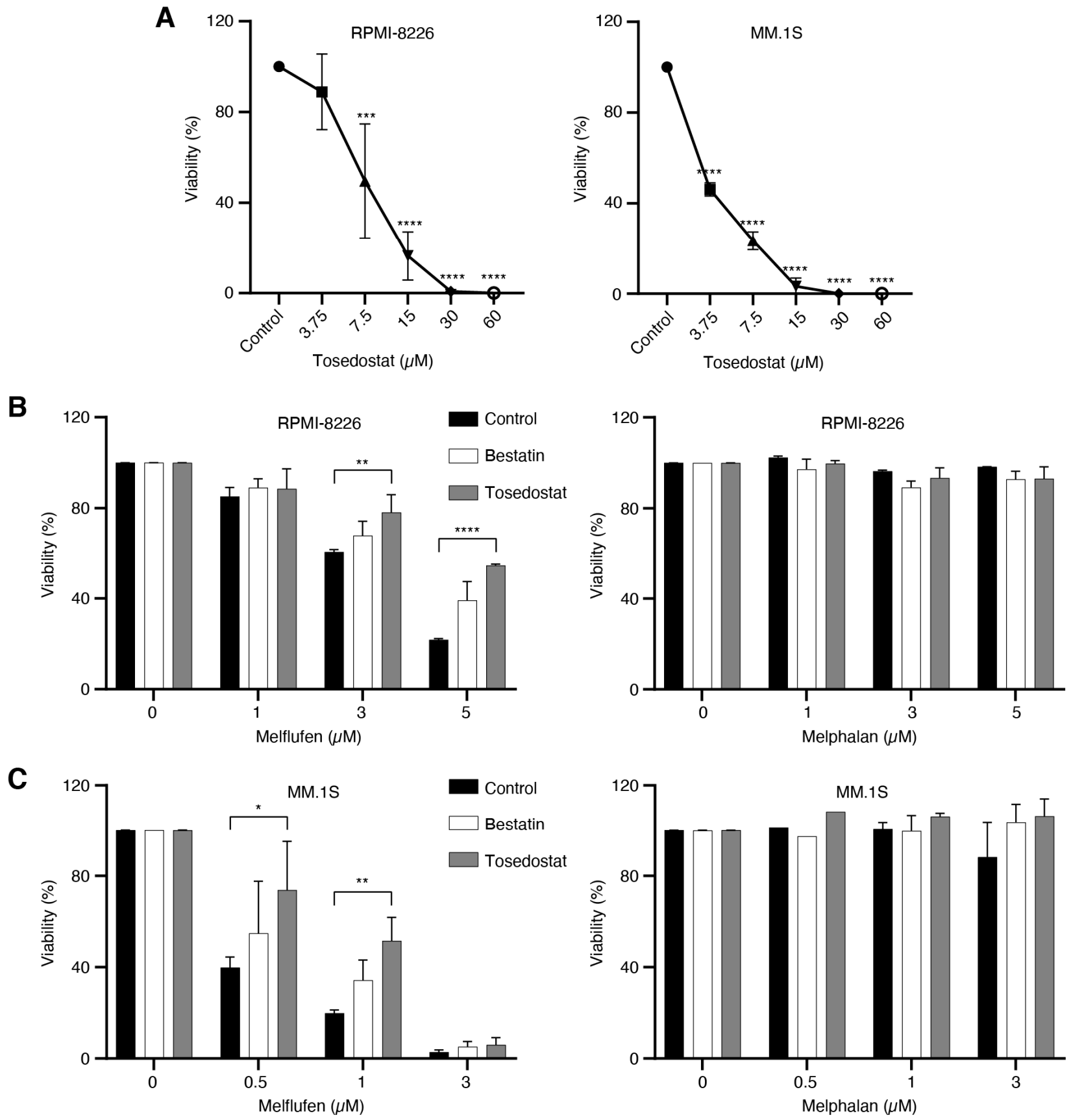

Figure 5. Aminopeptidase inhibitors reduce sensitivity to melflufen in MM cell lines. (A) MM cell lines RPMI-8226 and MM.1S were treated with different concentrations of the aminopeptidase inhibitor tosedostat for $48 \mathrm{~h}$, and cell viability was measured using PrestoBlue cell viability reagent. (B) RPMI-8226 cells or (C) MM.1S cells were pretreated with DMSO (control) or the aminopeptidase inhibitors bestatin $(10 \mu \mathrm{M})$ or tosedostat $(10 \mu \mathrm{M})$ for $1 \mathrm{~h}$, then either left without additional treatment or treated with three different concentrations of melflufen or melphalan for $15 \mathrm{~min}$, after which the medium was replaced. After $48 \mathrm{~h}$, cell viability was measured using PrestoBlue cell viability reagent. Statistical significance is indicated as ${ }^{*} p \leq 0.05 ;{ }^{* *} p \leq 0.01 ;{ }^{* * *} p \leq 0.001 ;{ }^{* * *} p \leq 0.0001$. DMSO: dimethyl sulfoxide 
A<smiles>CCOC(=O)C(N)Cc1ccc(F)cc1CC(NC(=O)C(N)Cc1ccc(N(CCCl)CCCl)cc1)C(=O)NC(Cc1ccc(F)cc1)C(=O)OCC</smiles>

B

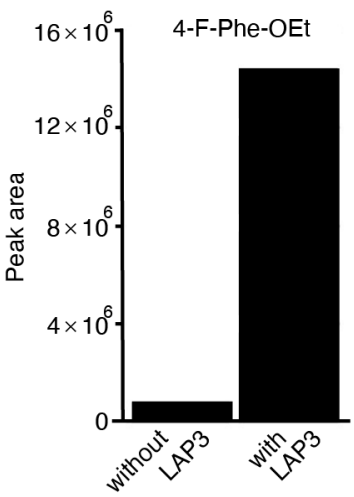

C

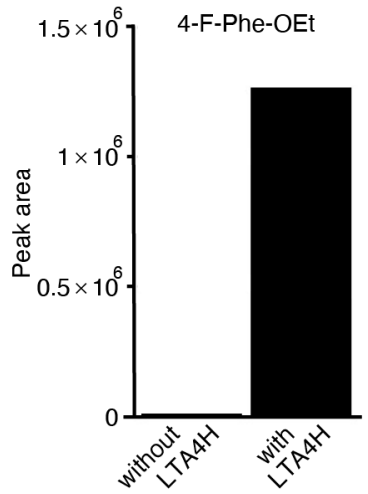

D



Figure 6. Aminopeptidases LAP3, LTA4H, and RNPEP can hydrolyze melflufen to melphalan and 4-F-Phe-OEt. (A) Reaction pathway of the hydrolysis of melflufen peptide bond by the aminopeptidases LAP3, LTA4H, and RNPEP. (B-D) Incubation buffer and substrates were mixed and incubated without and with (B) LAP3, (C) LTA4H, and (D) RNPEP for $2 \mathrm{~h}$ at $37^{\circ} \mathrm{C}$ (with duplicate incubations). The incubation was stopped by adding ice-cold acetonitrile. Using LC-HRMS, 4-F-Phe-OEt was then quantified, with concentrations presented as the peak area in the figure. 4-F-Phe-OEt: para-fluoro-L-phenylalanine ethyl ester; LC-HRMS: liquid chromatography-high-resolution mass spectrometry.

\section{Discussion}

Aminopeptidases are ubiquitous enzymes with important roles in cell development, growth, and maintenance. They are widely expressed in tissues, including hematopoietic cells of the bone marrow and peripheral blood, and in lymphoid organs $[27,28]$. Aminopeptidases are thought to have basic roles in regulating cell homeostasis, but more specific functions have recently been identified, particularly in malignancies [1,29]. The utilization of aminopeptidases for the processing of novel therapeutic drugs, such as antibody-drug conjugates and peptide-drug conjugates, is an active research area in drug development, especially for plasma cell diseases. The chemical linker in melflufen is made of a dipeptide, which is a substrate for aminopeptidases, resulting in hydrolysis and rapid release of the cytotoxic alkylator payload and intermediate metabolites [30]. Therefore, the expression and activity of aminopeptidases in MM cells is of particular interest.

Analysis of gene expression profiles of CD138+ cells from MM patient samples from two datasets showed that the majority of aminopeptidases are expressed at relatively similar levels. LAP3, one of the most highly expressed aminopeptidases in MM, is an enzyme that primarily catalyzes the hydrolysis of leucine residues from the amino-termini of proteins or peptides [31]. LAP3 is also implicated in antigen and major histocompatibility complex class I peptide processing, and is associated with the proinflammatory effects of IFN- $\gamma$ [32]. In cancer, LAP3 has also been linked to tumor cell proliferation, migration, and invasion $[33,34]$. Altered LAP3 expression has also been observed in esophageal squamous cell and hepatocellular carcinoma $[35,36]$. The role of LAP3 in plasma cells and other hematological cells is currently not known. 
Only five aminopeptidases, namely, ERAP2, XPNPEP1, DPP3, RNPEP, and CTSV, were differentially expressed between NDMM and RRMM. In the endoplasmic reticulum, ERAP enzymes trim peptides for presentation on major histocompatibility complex class I molecules [37]. A small number of studies demonstrate the direct involvement of ERAP enzymes in tumor growth and the generation of tumor epitopes for the presentation of cytotoxic T cells [38]. For example, the absence of ERAP2 in choriocarcinoma has been linked to the reduction of the activation of T lymphocytes by the tumor cells [39]. One could speculate that the reduction of ERAP2 expression in myeloma cells could be beneficial by reducing their immunogenicity and leading to their increased survival by immune evasion. The selection for lower ERAP2-expressing cells would naturally occur during disease progression. Another hypothetical link between MM and ERAP2 could be the excessive plasma protein production in MM cells that induces endoplasmic reticulum stress. The proteasomal degradation of the paraprotein is required to avoid endoplasmic reticulum stress-induced cell death. If this machinery is inhibited or altered, it might lead to drug resistance. In addition, CTSV is a potential prognostic biomarker for progression with high CTSV expression shown to be associated with poor outcome in breast ductal carcinomas in situ [40].

We also found that elevated expression of the aminopeptidase genes XPNPEP1, RN$P E P, D P P 3$, and BLMH could predict poor survival of MM patients. The role of these and other highly expressed aminopeptidases in MM remains to be explored. Proline-specific DPPs are emerging drug targets, and DPP4 inhibitors (gliptins) are already approved globally for the treatment of diabetes. Inhibition of DPP7 leads to apoptosis of the majority of resting cells in chronic lymphocytic leukemia [41]. Another study found that inhibition of DPP7 leads to upregulation of $R B 1$ and $M Y C$ and decrease of $B C L 2$, leading to potential apoptosis induction [42]. However, TP53 inactivation prevents DPP7 downregulation [42]. Biallelic inactivation of TP53 is a hallmark of MM and could therefore explain DPP7 overexpression in MM [43,44]. DPP3 is overexpressed in estrogen receptor-positive breast cancer and correlates with poor prognosis, and it is also more highly expressed in endometrial carcinomas vs. normal tissue [45,46]. Off-target DPP8/9 inhibition in MM cells leads to apoptotic cell death signaling in the presence of DPP4 inhibitors (gliptins), whereas in AML the direct inhibition of DPP8/9 induces the pro-caspase-1-dependent inflammatory form of programmed cell death, pyroptosis [14,15].

There are only a very limited number of studies demonstrating the prognostic value of aminopeptidases, namely, BLMH, RNPEP, and XPNPEP1, in human tumors. BLMH may have multiple roles in different physiological and pathological conditions, such as resistance to bleomycin therapy in several cancer types, cellular detoxification, and training of peptides for antigen presentation [47,48]. The XPNPEP1 (alias aminopeptidase P) protein is specifically found in the blood vessels of lungs and also in lung tumors [49]. RNPEP (alias aminopeptidase B) mRNA levels have been demonstrated to be altered during colorectal adenoma-carcinoma evolution, and RNPEP plasma levels are also independent prognostic factors for colorectal cancer patients [50]. Both XPNPEP1 and RNPEP enzyme activities were found to be significantly elevated in thyroid neoplasms when compared with nonmalignant adjacent tissues [51].

Peptide-drug conjugates such as melflufen require aminopeptidases to unload their payload into cells. We found that melflufen could target CD138+CD38+ plasma cells in MM patient samples and was particularly active in RRMM samples. The higher expression of specific aminopeptidases in RRMM compared with NDMM could explain the differential activity of melflufen in samples from these two patient groups. However, from our limited set of samples, we did not observe significant correlation between melflufen sensitivity and expression of an individual aminopeptidase gene. Nevertheless, hydrolysis analysis of seven aminopeptidases demonstrated that LAP3, LTA4H, RNPEP, DPP7, and ANPEP are able to hydrolyze melflufen. In addition, we demonstrated that the efficacy of melflufen, but not melphalan, could be reduced with the addition of the metalloaminopeptidase 
inhibitors tosedostat or bestatin in MM cells lines. This indicates a difference in the mode of action between melphalan and melflufen.

Melflufen is being investigated in several clinical trials. The first study reported shows that melflufen combined with dexamethasone leads to clinical improvement with an overall response rate of $31 \%$, median progression-free survival of 5.7 months, and median overall survival of 20.7 months [52]. It also demonstrated that melflufen is equally effective in melphalan-naïve and melphalan-refractory patients [52]. The second study, focusing on heavily pretreated RRMM, showed that melflufen combined with dexamethasone leads to clinical improvement in the triple-class RRMM population with an overall response rate of $26 \%$, median progression-free survival of 3.9 months, and median overall survival of 11.2 months [53]. In this study, we demonstrated that melflufen is highly effective at targeting plasma cells, particularly in samples from RRMM patients, including those with poor prognostic markers, such as del $17 \mathrm{p}$ or $\mathrm{t}(4 ; 14)$. We also showed that melflufen can be hydrolyzed to its active form by aminopeptidases that are highly expressed in MM. Although additional investigations are needed to understand the specific role of aminopeptidases in MM pathogenesis, our results highlight that this group of enzymes can be exploited to benefit MM patients who may not respond to other available therapies.

\section{Conclusions}

In this study we have analyzed the expression of aminopeptidase genes in MM patients and how aminopeptidase gene expression correlates with patient overall survival. We also tested the sensitivity of MM plasma cells to peptide-drug conjugate melflufen, which is known to be activated by aminopeptidases, and how inhibition of aminopeptidases affect melflufen activity in MM cell lines. Based on ranked abundance, LAP3, ERAP2, METAP2, TTP2, and DPP7 were highly expressed in MM. ERAP2, XPNPEP1, DPP3, RNPEP, and CTS $V$ were differentially expressed between RRMM and NDMM samples $(p<0.05)$. Survival analysis revealed that high expression of XPNPEP1, RNPEP, DPP3, and BLMH $(p<0.05)$ was associated with shorter overall survival. Sensitivity to melflufen was detected ex vivo in the majority of MM patient samples, and high sensitivity was observed, especially in RRMM samples. Hydrolysis analysis demonstrated that melflufen is a substrate for aminopeptidases LAP3, LTA4H, RNPEP, and ANPEP, which are expressed in MM. The sensitivity of MM cell lines to melflufen was reduced by aminopeptidase inhibitors. These results indicate critical roles of aminopeptidases in disease progression and the activity of melflufen in MM.

Supplementary Materials: The following are available online at https: / www.mdpi.com/article / 10.3390/cancers13071527/s1: Figure S1: Drug plate layout and drug concentrations used in the flow cytometry-based drug sensitivity testing, Figure S2: Flow cytometry gating strategy used in drug sensitivity testing, Figure S3: Aminopeptidase gene family genes showed similar expression in both the CoMMpass and FIMM datasets, Figure S4: Correlation of aminopeptidase genes LAP3, ERAP2, METAP2, TPP2, DPP7, ERAP1, LTA4H, and LNPEP (Group I) expression with myeloma patient cytogenetics and age in the FIMM dataset, Figure S5: Aminopeptidase gene expression positively correlates with aminopeptidase protein expression, especially for LAP3, BLMH, DPP3, DPP7, RNPEP, and ERAP2 aminopeptidases, Figure S6: CoMMpass dataset confirms that aminopeptidase genes are differentially expressed in NDMM vs. RRMM, Figure S7: Correlation of aminopeptidase genes differentially expressed between RRMM and NDMM with myeloma patient cytogenetics and ISS stage in the FIMM dataset, Figure S8: Prognostic significance of three aminopeptidase genes $X P N P E P 1, R N P E P$, and DPP3 expression in NDMM and RRMM samples separately in the FIMM dataset, Figure S9: Prognostic significance of aminopeptidase gene expression in the CoMMpass dataset, Figure S10: Somatic mutation predictions for aminopeptidase genes in the FIMM dataset samples $(n=169)$, Figure S11: Somatic mutation predictions for aminopeptidase genes in CoMMpass dataset $(n=1164)$, Figure S12: Aminopeptidase gene CNVs in FIMM dataset MM samples $(n=169)$, Figure S13: Aminopeptidase gene CNVs in CoMMpass dataset $(n=1044)$, Figure S14: Ex vivo sensitivity of MM CD138+CD38+ plasma cells to melflufen, melphalan, bortezomib, selinexor, and 4-HC, Figure S15: Ex vivo sensitivity of MM CD138+CD38+ plasma cells to melflufen in $10 \mathrm{MM}$ 
patients with gene expression data also available from MM CD138+ plasma cells., Figure S16: Correlation of the 39 aminopeptidase genes' expression with melflufen sensitivity in bone marrow plasma cells in 10 myeloma patient samples, Figure S17: Correlation of drug sensitivity (EC50 values) to selinexor vs. melflufen, melphalan, and bortezomib in CD138+CD38+ plasma cells from MM patient samples, Figure S18: Cytogenetics and melflufen sensitivity in CD138+CD38+ plasma cells from MM patient samples, Figure S19: Aminopeptidase ANPEP can hydrolyze melflufen to melphalan and 4-F-Phe-OEt, Table S1: FIMM dataset patient sample characteristics and sample analysis information, Table S2: List of the 39 annotated aminopeptidase genes in the human genome utilizing the Ensembl and NCBI databases and further confirming the molecular function (gene ontology) of the identified genes, Table S3: List of compounds used in the experiments, Table S4: Aminopeptidases used in the hydrolysis assay and their incubation buffers, Table S5: Log2(RPKM) expression values for 39 aminopeptidase genes and 17 housekeeping genes in MM patient samples in the FIMM dataset $(n=122)$, Table S6: LC-MS/MS-based proteomics label-free quantitation intensity values for 17 aminopeptidase proteins in CD138+ cells isolated from MM patient samples in the FIMM dataset $(n=23)$, Table S7: Statistical overview of genes identified as prognostic markers $(p \leq 0.05)$ in the FIMM dataset $(n=122)$, Table S8: Aminopeptidase gene somatic mutation frequencies from MM patient samples in the FIMM dataset $(n=169)$, Table S9: Aminopeptidase gene copy number variation scores from MM patient samples in the FIMM dataset $(n=169)$, Table S10: Summary of live cells and CD138+CD38+ plasma cells present in the BM-MNC samples after $72 \mathrm{~h}$ incubation in DMSO (control), Table S11: Melflufen, melphalan, selinexor, bortezomib, and 4-HC EC50 values from 15 MM patient samples' CD138+CD38+ plasma cells with sample disease stage indicated, Table S12: Mean $\log 2(\mathrm{RPKM})$ values for the 39 aminopeptidase genes in melflufen high-sensitivity $(n=5)$ and low-sensitivity $(n=5)$ myeloma samples.

Author Contributions: Conceptualization, J.J.M., R.K., N.N.N., F.L. and C.A.H.; data curation, J.J.M. and J.L.; formal analysis, J.J.M., R.K., G.A.T., P.S., M.M.M., A.S. (Alexander Schepsky), D.B., P.D. and A.S. (Ana Slipicevic); funding acquisition, C.A.H.; methodology, J.J.M., R.K., G.A.T., M.-E.H., P.S., M.M.M., A.S. (Alexander Schepsky) and P.D.; project administration, C.A.H.; resources, J.L., P.A. and R.S.; supervision, T.G., P.O., A.S. (Ana Slipicevic), N.N.N., F.L. and C.A.H.; visualization, J.J.M., R.K., G.A.T., P.S., and A.S. (Ana Slipicevic); writing-original draft, J.J.M., R.K., N.N.N. and C.A.H.; writing-review and editing, J.J.M., R.K., G.A.T., M.-E.H., P.S., M.M.M., A.S. (Alexander Schepsky), T.G., J.L., D.B., P.D., P.O., A.S. (Ana Slipicevic), P.A., R.S., N.N.N., F.L. and C.A.H. All authors have read and agreed to the published version of the manuscript.

Funding: This work has been supported by funding from Oncopeptides AB, Academy of Finland (grant 1320185), Cancer Society of Finland, and Sigrid Jusélius Foundation.

Institutional Review Board Statement: The study was conducted according to the guidelines of the Declaration of Helsinki, and approved by an ethical committee of the Helsinki University Hospital (permit numbers 239/13/03/00/2010 and 303/13/03/01/2011, date of approval, 5.11.2012, and approval date for project extension, 13.2.2018)

Informed Consent Statement: Written informed consent has been obtained from the patients to collect patient samples and to publish the data used in this paper.

Data Availability Statement: The data presented in this study are available on request from the corresponding author. The data are not publicly available due to privacy and ethical limitations.

Acknowledgments: These data were generated in part by the Multiple Myeloma Research Foundation Personalized Medicine Initiatives (https://research.themmrf.org and www.themmrf.org, accessed on 5 February 2018). This work has been supported by funding from Oncopeptides AB, Academy of Finland (grant 1320185), Cancer Society of Finland, and Sigrid Jusélius Foundation. The authors acknowledge use of the Q-Exactive quantitative mass spectrometer, funded under the Research Infrastructure Call 2012 by Science Foundation Ireland (SFI-12/RI/2346/3). The authors would like to thank Alun Parsons, Minna Suvela, the FIMM Sequencing Unit, the FIMM High Throughput Biomedicine Unit, and the FIMM Bioinformatics Unit for their excellent technical support. A\&M Labor GmbH (Bergheim, Germany) is acknowledged for performing the aminopeptidase hydrolysis assay experiments. The authors are very grateful to the patients who generously donated samples for our research. The authors would also like to thank the Finnish Hematology Research Biobank (FHRB; Helsinki, Finland) for providing the viably frozen samples for the flow cytometry-based drug sensitivity testing experiments. 
Conflicts of Interest: All authors met the criteria set forth by the International Committee of Medical Journal Editors (ICMJE) and hence adequately contributed to manuscript development. A.S. and F.L. are employees of Oncopeptides AB. N.N.N. is a consultant for Oncopeptides AB. C.A.H. has received research funding from Oncopeptides AB, Kronos Bio, Novartis, Celgene, Orion Pharma, and the IMI2 consortium project HARMONY. T.G., G.A.T., and A.S. have a research contract with Oncopeptides AB. R.S. has received research funding from Amgen, BMS, Celgene, and Takeda and honoraria from Amgen, Celgene, Janssen-Cilag, Takeda, and Sanofi. J.L. and P.A. have received personal fees from Amgen, Bristol Myers Squibb, Celgene, Janssen, Sanofi, and Takeda. J.J.M., R.K., M.E.H., P.S., M.M.M., D.B., P.D., and P.O. declare no conflicts of interest.

\section{References}

1. Moore, H.E.; Davenport, E.L.; Smith, E.M.; Muralikrishnan, S.; Dunlop, A.S.; Walker, B.A.; Krige, D.; Drummond, A.H.; Hooftman, L.; Morgan, G.J.; et al. Aminopeptidase inhibition as a targeted treatment strategy in myeloma. Mol. Cancer Ther. 2009, 8, 762-770. [CrossRef]

2. Leleu, X.; Martin, T.G.; Einsele, H.; Lyons, R.M.; Durie, B.G.; Iskander, K.S.; Ailawadhi, S. Role of Proteasome Inhibitors in Relapsed and/or Refractory Multiple Myeloma. Clin. Lymphoma Myeloma Leuk. 2019, 19, 9-22. [CrossRef]

3. Sanderink, G.-J.; Artur, Y.; Siest, G. Human Aminopeptidases: A Review of the Literature. Clin. Chem. Lab. Med. 1988, 26, 795-807. [CrossRef] [PubMed]

4. Ackerman, G.A. Histochemical demonstration of aminopeptidase activity in the leukocytes of blood and bone marrow. J. Histochem. Cytochem. 1960, 8, 386. [CrossRef]

5. Ackerman, G.A. Histochemical Demonstration of Aminopeptidase Activity in the Cells of the Blood and Bone Marrow from Various Hæmatological Disorders. Nat. Cell Biol. 1963, 197, 189-190. [CrossRef] [PubMed]

6. Dalal, B.I.; Al Mugairi, A.; Pi, S.; Lee, S.Y.; Khare, N.S.; Pal, J.; Bryant, A.; Vakil, A.P.; Lau, S.; Mourad, Y.R.A. Aberrant Expression of CD13 Identifies a Subgroup of Standard-Risk Adult Acute Lymphoblastic Leukemia With Inferior Survival. Clin. Lymphoma Myeloma Leuk. 2014, 14, 239-244. [CrossRef] [PubMed]

7. Craddock, K.J.; Chen, Y.; Brandwein, J.M.; Chang, H. CD13 expression is an independent adverse prognostic factor in adults with Philadelphia chromosome negative B cell acute lymphoblastic leukemia. Leuk. Res. 2013, 37, 759-764. [CrossRef]

8. Lau, H.; Nagy, A.; Atwater, S.K.; Cascio, M.J.; Ohgami, R.S. An integrated flow cytometry analysis of 286 mature B cell neoplasms identifies CD13 as a useful marker for diagnostic subtyping. Int. J. Lab. Hematol. 2018, 40, 715-720. [CrossRef] [PubMed]

9. García-Sanz, R.; Orfão, A.; González, M.; Tabernero, M.; Bladé, J.; Moro, M.; Fernández-Calvo, J.; Sanz, M.; Pérez-Simoón, J.; Rasillo, A.; et al. Primary Plasma Cell Leukemia: Clinical, Immunophenotypic, DNA Ploidy, and Cytogenetic Characteristics. Blood 1999, 93, 1032-1037. [CrossRef] [PubMed]

10. Raimbault, A.; Machherndl-Spandl, S.; Itzykson, R.; Clauser, S.; Chapuis, N.; Mathis, S.; Lauf, J.; Alary, A.-S.; Burroni, B.; Kosmider, O.; et al. CD13 expression in B cell malignancies is a hallmark of plasmacytic differentiation. Br. J. Haematol. 2019, 184, 625-633. [CrossRef]

11. Shim, H.; Ha, J.H.; Lee, H.; Sohn, J.Y.; Kim, H.J.; Eom, H.-S.; Kong, S.-Y. Expression of Myeloid Antigen in Neoplastic Plasma Cells Is Related to Adverse Prognosis in Patients with Multiple Myeloma. BioMed Res. Int. 2014, 2014, 1-8. [CrossRef]

12. Kakodkar, P.; More, S.; András, K.; Papakonstantinou, N.; Kelly, S.; Makrooni, M.A.; Ortutay, C.; Szegezdi, E. Aspartic Aminopeptidase Is a Novel Biomarker of Aggressive Chronic Lymphocytic Leukemia. Cancers 2020, 12, 1876. [CrossRef] [PubMed]

13. Lim, Y.W.; Chen-Harris, H.; Mayba, O.; Lianoglou, S.; Wuster, A.; Bhangale, T.; Khan, Z.; Mariathasan, S.; Daemen, A.; Reeder, J.; et al. Germline genetic polymorphisms influence tumor gene expression and immune cell infiltration. Proc. Natl. Acad. Sci. USA 2018, 115, E11701-E11710. [CrossRef] [PubMed]

14. Sato, T.; Tatekoshi, A.; Takada, K.; Iyama, S.; Kamihara, Y.; Jawaid, P.; Rehman, M.U.; Noguchi, K.; Kondo, T.; Kajikawa, S.; et al. DPP8 is a novel therapeutic target for multiple myeloma. Sci. Rep. 2019, 9, 1-8. [CrossRef]

15. Johnson, D.C.; Taabazuing, C.Y.; Okondo, M.C.; Chui, A.J.; Rao, S.D.; Brown, F.C.; Reed, C.; Peguero, E.; De Stanchina, E.; Kentsis, A.; et al. DPP8/DPP9 inhibitor-induced pyroptosis for treatment of acute myeloid leukemia. Nat. Med. 2018, 24, 1151-1156. [CrossRef]

16. Dinardo, C.D.; Cortes, J.E. Tosedostat for the treatment of relapsed and refractory acute myeloid leukemia. Expert Opin. Investig. Drugs 2013, 23, 265-272. [CrossRef] [PubMed]

17. Mawad, R.; Becker, P.S.; Hendrie, P.; Scott, B.; Wood, B.L.; Dean, C.; Sandhu, V.; Deeg, H.J.; Walter, R.; Wang, L.; et al. Phase II study of tosedostat with cytarabine or decitabine in newly diagnosed older patients with acute myeloid leukaemia or high-risk MDS. Br. J. Haematol. 2015, 172, 238-245. [CrossRef]

18. Cohen, A.D. Myeloma: Next generation immunotherapy. Hematology 2019, 2019, 266-272. [CrossRef]

19. Gullbo, J.; Wickström, M.; Tullberg, M.; Ehrsson, H.; Lewensohn, R.; Nygren, P.; Luthman, K.; Larsson, R. Activity of Hydrolytic Enzymes in Tumour Cells is a Determinant for Anti-tumour Efficacy of the Melphalan Containing Prodrug J1. J. Drug Target. 2003, 11, 355-363. [CrossRef] 
20. Chauhan, D.; Ray, A.; Viktorsson, K.; Spira, J.; Paba-Prada, C.; Munshi, N.; Richardson, P.; Lewensohn, R.; Anderson, K.C. In Vitro and In Vivo Antitumor Activity of a Novel Alkylating Agent, Melphalan-Flufenamide, against Multiple Myeloma Cells. Clin. Cancer Res. 2013, 19, 3019-3031. [CrossRef] [PubMed]

21. Ray, A.; Ravillah, D.; Das, D.S.; Song, Y.; Nordström, E.; Gullbo, J.; Richardson, P.G.; Chauhan, D.; Anderson, K.C. A novel alkylating agent Melflufen induces irreversible DNA damage and cytotoxicity in multiple myeloma cells. Br. J. Haematol. 2016, 174, 397-409. [CrossRef]

22. Dobin, A.; Davis, C.A.; Schlesinger, F.; Drenkow, J.; Zaleski, C.; Jha, S.; Batut, P.; Chaisson, M.; Gingeras, T.R. STAR: Ultrafast universal RNA-seq aligner. Bioinformatics 2013, 29, 15-21. [CrossRef]

23. Love, M.I.; Huber, W.; Anders, S. Moderated estimation of fold change and dispersion for RNA-seq data with DESeq2. Genome Biol. 2014, 15, 550. [CrossRef]

24. Koboldt, D.C.; Zhang, Q.; Larson, D.E.; Shen, D.; McLellan, M.D.; Lin, L.; Miller, C.A.; Mardis, E.R.; Ding, L.; Wilson, R.K. VarScan 2: Somatic mutation and copy number alteration discovery in cancer by exome sequencing. Genome Res. 2012, 22, 568-576. [CrossRef]

25. Cingolani, P.; Platts, A.; Wang, L.L.; Coon, M.; Nguyen, T.; Wang, L.; Land, S.J.; Lu, X.; Ruden, D.M. A program for annotating and predicting the effects of single nucleotide polymorphisms, SnpEff: SNPs in the genome of Drosophila melanogaster strain w1118; iso-2; iso-3. Fly 2012, 6, 80-92. [CrossRef]

26. Caers, J.; Garderet, L.; Kortüm, K.M.; O’Dwyer, M.E.; Van De Donk, N.W.; Binder, M.; Dold, S.M.; Gay, F.; Corre, J.; Beguin, Y.; et al. European Myeloma Network recommendations on tools for the diagnosis and monitoring of multiple myeloma: What to use and when. Haematology 2018, 103, 1772-1784. [CrossRef]

27. Razak, K.; Newland, A. The significance of aminopeptidases and haematopoietic cell differentiation. Blood Rev. 1992, 6, 243-250. [CrossRef]

28. Uhlén, M.; Fagerberg, L.; Hallström, B.M.; Lindskog, C.; Oksvold, P.; Mardinoglu, A.; Sivertsson, Å.; Kampf, C.; Sjöstedt, E.; Asplund, A.; et al. Tissue-based map of the human proteome. Science 2015, 347, 1260419. [CrossRef] [PubMed]

29. Compagnone, M.; Cifaldi, L.; Fruci, D. Regulation of ERAP1 and ERAP2 genes and their disfunction in human cancer. Hum. Immunol. 2019, 80, 318-324. [CrossRef] [PubMed]

30. Wickström, M.; Nygren, P.; Larsson, R.; Harmenberg, J.; Lindberg, J.; Sjöberg, P.; Jerling, M.; Lehmann, F.; Richardson, P.; Anderson, K.; et al. Melflufen-A peptidase-potentiated alkylating agent in clinical trials. Oncotarget 2017, 8, 66641-66655. [CrossRef]

31. Matsui, M.; Fowler, J.H.; Walling, L.L. Leucine aminopeptidases: Diversity in structure and function. Biol. Chem. 2006, 387, 1535-1544. [CrossRef] [PubMed]

32. Beninga, J.; Rock, K.L.; Goldberg, A.L. Interferon- $\gamma$ Can Stimulate Post-proteasomal Trimming of the N Terminus of an Antigenic Peptide by Inducing Leucine Aminopeptidase. J. Biol. Chem. 1998, 273, 18734-18742. [CrossRef] [PubMed]

33. Fang, C.; Zhang, J.; Yang, H.; Peng, L.; Wang, K.; Wang, Y.; Zhao, X.; Liu, H.; Dou, C.; Shi, L.; et al. Leucine aminopeptidase 3 promotes migration and invasion of breast cancer cells through upregulation of fascin and matrix metalloproteinases-2/9 expression. J. Cell. Biochem. 2019, 120, 3611-3620. [CrossRef] [PubMed]

34. He, X.; Huang, Q.; Qiu, X.; Liu, X.; Sun, G.; Guo, J.; Ding, Z.; Yang, L.; Ban, N.; Tao, T.; et al. LAP3 promotes glioma progression by regulating proliferation, migration and invasion of glioma cells. Int. J. Biol. Macromol. 2015, 72, 1081-1089. [CrossRef] [PubMed]

35. Tian, S.-Y.; Chen, S.-H.; Shao, B.-F.; Cai, H.-Y.; Zhou, Y.; Zhou, Y.-L.; Xu, A.-B. Expression of leucine aminopeptidase 3 (LAP3) correlates with prognosis and malignant development of human hepatocellular carcinoma (HCC). Int. J. Clin. Exp. Pathol. 2014, 7, 3752-3762.

36. Zhang, S.; Yang, X.; Shi, H.; Li, M.; Xue, Q.; Ren, H.; Yao, L.; Chen, X.; Zhang, J.; Wang, H. Overexpression of leucine aminopeptidase 3 contributes to malignant development of human esophageal squamous cell carcinoma. J. Mol. Histol. 2014, 45, 283-292. [CrossRef]

37. Hattori, A.; Tsujimoto, M. Endoplasmic reticulum aminopeptidases: Biochemistry, physiology and pathology. J. Biochem. 2013, 154, 219-228. [CrossRef] [PubMed]

38. Stratikos, E. Modulating antigen processing for cancer immunotherapy. OncoImmunology 2014, 3, e27568. [CrossRef] [PubMed]

39. Warthan, M.D.; Washington, S.L.; Franzese, S.E.; Ramus, R.M.; Kim, K.-R.; York, T.P.; Stratikos, E.; Strauss, J.F.; Lee, E.D. The role of endoplasmic reticulum aminopeptidase 2 in modulating immune detection of choriocarcinomat. Biol. Reprod. 2018, 98, 309-322. [CrossRef]

40. Toss, M.; Miligy, I.; Gorringe, K.; Mittal, K.; Aneja, R.; Ellis, I.; Green, A.; Rakha, E. Prognostic significance of cathepsin V (CTSV/CTSL2) in breast ductal carcinoma in situ. J. Clin. Pathol. 2019, 73, 76-82. [CrossRef]

41. Danilov, A.V.; Danilova, O.V.; Brown, J.R.; Rabinowitz, A.; Klein, A.K.; Huber, B.T. Dipeptidyl peptidase 2 apoptosis assay determines the B-cell activation stage and predicts prognosis in chronic lymphocytic leukemia. Exp. Hematol. 2010, 38, 1167-1177. [CrossRef] [PubMed]

42. Mele, D.A.; Bista, P.; Baez, D.V.; Huber, B.T. Dipeptidyl Peptidase 2 is an essential survival factor in the regulation of cell quiescence. Cell Cycle 2009, 8, 2425-2434. [CrossRef]

43. Shah, V.; Johnson, D.C.; Sherborne, A.L.; Ellis, S.; Aldridge, F.M.; Howard-Reeves, J.; Begum, F.; Price, A.; Kendall, J.; Chiecchio, L.; et al. Subclonal TP53 copy number is associated with prognosis in multiple myeloma. Blood 2018, 132, 2465-2469. [CrossRef] 
44. Thakurta, A.; Ortiz, M.; Blecua, P.; Towfic, F.; Corre, J.; Serbina, N.V.; Flynt, E.; Yu, Z.; Yang, Z.; Palumbo, A.; et al. High subclonal fraction of 17p deletion is associated with poor prognosis in multiple myeloma. Blood 2019, 133, 1217-1221. [CrossRef] [PubMed]

45. Lu, K.; Alcivar, A.L.; Ma, J.; Foo, T.K.; Zywea, S.; Mahdi, A.; Huo, Y.; Kensler, T.W.; Gatza, M.L.; Xia, B. NRF2 Induction Supporting Breast Cancer Cell Survival Is Enabled by Oxidative Stress-Induced DPP3-KEAP1 Interaction. Cancer Res. 2017, 77, 2881-2892. [CrossRef] [PubMed]

46. Šimaga, Š.; Babić, D.; Osmak, M.; Ilić-Forko, J.; Vitale, L.; Miličić, D.; Abramić, M. Dipeptidyl peptidase III in malignant and non-malignant gynaecological tissue. Eur. J. Cancer 1998, 34, 399-405. [CrossRef]

47. Chen, Y.; Zhang, H.; He, Q. Involvement of bleomycin hydrolase and poly(ADP-ribose) polymerase-1 in Ubc9-mediated resistance to chemotherapy agents. Int. J. Oncol. 2016, 50, 223-231. [CrossRef] [PubMed]

48. Jóna, Á.; Miltenyi, Z.; Póliska, S.; Bálint, B.L.; Illes, A. Effect of Bleomycin Hydrolase Gene Polymorphism on Late Pulmonary Complications of Treatment for Hodgkin Lymphoma. PLoS ONE 2016, 11, e0157651. [CrossRef] [PubMed]

49. Oh, P.; Li, Y.; Yu, J.; Durr, E.; Krasinska, K.M.; Carver, L.A.; Testa, J.E.; Schnitzer, J.E. Subtractive proteomic mapping of the endothelial surface in lung and solid tumours for tissue-specific therapy. Nat. Cell Biol. 2004, 429, 629-635. [CrossRef] [PubMed]

50. Perez, I.; Blanco, L.; Sanz, B.; Errarte, P.; Ariz, U.; Beitia, M.; Fernández, A.; Loizate, A.; Candenas, M.L.; Pinto, F.M.; et al. Altered Activity and Expression of Cytosolic Peptidases in Colorectal Cancer. Int. J. Med Sci. 2015, 12, 458-467. [CrossRef]

51. Larrinaga, G.; Blanco, L.; Errarte, P.; Beitia, M.; Sanz, B.; Perez, I.; Irazusta, A.; Sánchez, C.E.; Santaolalla, F.; Andrés, L.; et al. Altered Peptidase Activities in Thyroid Neoplasia and Hyperplasia. Dis. Markers 2013, 35, 825-832. [CrossRef] [PubMed]

52. Richardson, P.G.; Bringhen, S.; Voorhees, P.; Plesner, T.; Mellqvist, U.-H.; Reeves, B.; Paba-Prada, C.; Zubair, H.; Byrne, C.; Chauhan, D.; et al. Melflufen plus dexamethasone in relapsed and refractory multiple myeloma (O-12-M1): A multicentre, international, open-label, phase 1-2 study. Lancet Haematol. 2020, 7, e395-e407. [CrossRef]

53. Richardson, P.G.; Oriol, A.; Larocca, A.; Bladé, J.; Cavo, M.; Rodriguez-Otero, P.; Leleu, X.; Nadeem, O.; Hiemenz, J.W.; Hassoun, H.; et al. Melflufen and Dexamethasone in Heavily Pretreated Relapsed and Refractory Multiple Myeloma. J. Clin. Oncol. 2021, 39, 757-767. [CrossRef] [PubMed] 